\title{
Effects of attention manipulation on judgments of duration and of intensity in the visual modality
}

\author{
LAURENCE CASINI and FRANÇOISE MACAR \\ Centre de Recherche en Neurosciences Cognitives, Équipe Temps \\ CNRS, Marseille, France
}

\begin{abstract}
The "attentional model" of time estimation assumes that temporal judgments depend on the amount of attention allocated to the temporal processor (the timer). One of the main predictions of this model is that an interval will be judged shorter when attention is not allocated to the temporal parameters of the task. Previous studies combining temporal and nontemporal tasks (dual-task method) have suggested that the time spent processing the target duration might be a key factor: The less time devoted by the subject to the temporal task, the shorter the judged duration. In the two experiments presented here, subjects were asked to judge both the duration of a visual stimulus and an increment in intensity occurring at any time during this stimulus. In the second experiment, trials without intensity increments were added. The main result is that the judged duration was shorter when the increment occurred later in the stimulus or did not occur. In those cases, subjects had been expecting increment occurrence during most part of the stimulus and thus had focused for a shorter time on stimulus duration. We propose that attention shifts related to expectancy and to detection of the increment reduce subjective duration.
\end{abstract}

Dual-task paradigms have been extensively used to demonstrate that judged durations in the second-tominute range are inversely correlated to the amount of attention paid to the elapsing time. In such dual-task paradigms, subjects have to perform two tasks simultaneously, one temporal (estimation of the duration of a stimulus) and the other nontemporal (e.g., judgment of the intensity, frequency, or brightness of a stimulus). The amount of attention allocated to each task can be manipulated by controlling the quantity or the complexity of nontemporal information. The idea is that more attentional resources are required by the nontemporal task when it is more complex or longer to perform. When this occurs, the main consequence is that estimated duration becomes shorter (Hicks, Miller, \& Kinsbourne, 1976; McClain, 1983; Zakay, 1989, 1992).

The dual-task studies provide arguments in favor of the attentional model of time perception first proposed by Thomas and Weaver (1975) for durations shorter than $100 \mathrm{msec}$ and developed by Zakay (1989) for longer durations. This model states that a stimulus is analyzed by two processors: a temporal processor, or "timer," whose output is a monotonic function of the duration of the stim-

The authors are grateful to Guy Reynard for developing the computer programs, to several colleagues from the Centre de Recherche en Neurosciences Cognitives, to Simon Grondin for seminal discussions on the data, and to Richard Ivry for his help with the English language and his critical comments on an earlier draft of this manuscript. Correspondence should be addressed to L. Casini or F. Macar. CNRS, CRNC, 31 chemin Joseph Aiguier, 13402 Marseille cedex 20, France (e-mail: casini@)!nf.cnrs-mrs.fr). ulus, and a processor of nontemporal information that encodes the nontemporal characteristics of the stimulus. Attentional resources would be divided between all the tasks that an organism has to perform and, hence, between those processors. The temporal processor is assumed to accumulate "subjective time units" (Zakay, 1989 ) or "pulses" emitted by a pacemaker (Creelman, 1962; Gibbon, Church, \& Meck, 1984; Treisman, 1963). Admittedly, the nature of these basic elements is entirely speculative; the oscillatory properties of certain cells or neuronal networks have been proposed to play this role (Miall, 1992; Treisman, 1993).

The number of accumulated pulses should increase with the target duration under "prospective" conditions (i.e., when the subject is forewarned that a temporal judgment will be required), because priority is given to the temporal task. Nevertheless, if the nontemporal task is complex, it will require attentional resources and thus will impinge on the resources available for the timer. This is expected to reduce the number of accumulated pulses. As a consequence, a negative relation should appear between perceived time and the complexity of a nontemporal secondary task. Therefore, when a temporal task and a nontemporal task are simultaneously performed, the attentional model yields two predictions: First, estimated duration should shorten when the amount of attention devoted to the temporal task decreases, and, second, interference effects should exist between the tasks, if both draw on the same pool of resources.

To explore the role of attention in temporal estimation, we tested the view that attentional resources may be divided between temporal information (duration of a stimu- 
lus) and nontemporal information (intensity of a stimulus) by using dual-task paradigms with explicit instructions to subjects on how to allocate their attention between both tasks (Casini, Macar, \& Grondin, 1992; Grondin \& Macar, 1992; Macar, Grondin, \& Casini, 1994). This method contrasted with previous dual-task studies in which the attentional effects on subjective duration were inferred from the manipulation of the quantity and/or the complexity of nontemporal information. Both approaches rest on the assumption that attentional resources are limited and must be divided between tasks that are simultaneously performed. When more attention is allocated to one task, less attention is available for the other one, and this entails a tradeoff between the performances in each task. This tradeoff has been extensively analyzed in the field of visual attention (Kahneman, 1973; Navon, 1985; Navon \& Gopher, 1979). Sperling and Melchner (1978) discussed the nature of the attentional mechanisms that take place in divided attention situations. Attention can be either switched from one task to the other repeatedly during a trial or divided between the two tasks in fixed proportions during the entire trial. Switching mechanisms seems to be prevalent when the two tasks require more than a few milliseconds, which is the case here.

In our previous studies, both visual and auditory modalities were used, with durations ranging from 250 to $3,000 \mathrm{msec}$. The results showed that subjects could control their allocation of attentional resources to the two concurrent tasks. More interesting, the quantity of attention allocated to time was an important factor in temporal performance, with reduced attention associated with a loss of accuracy and a reduction in perceived duration. Our interpretation of the underestimation we observed is that the accumulation of pulses during the target duration was impaired each time attention was distracted from the timer. The duration or the number of attention shifts would determine the amount of lost pulses, and, hence the amount of temporal underestimation. The time spent processing intensity relative to duration could be the key factor, suggesting that the effect of attention occurred at an early stage - that is, when temporal information was generated by the timer.

The present experiments were a replication and an extension of our previous work (Macar et al., 1994) and were designed to analyze further the relation between temporal underestimation and attention shifts. Experiment 1 tested the hypothesis that a brief change in stimulus intensity occurring early or late in the course of the stimulus influences measures of sensitivity $\left(d^{\prime}\right.$ index) and of magnitude (proportion of durations judged "short") in the temporal task. The uncertainty about the occurrence of the change in intensity is supposed to require subjects to shift their attention repeatedly to the intensity until the increment occurs; thus, a large number of shifts (in the case of a late increment) should produce a larger shortening in subjective duration than should fewer shifts (early increment). Experiment 2 extended Experiment 1 by investigating whether the prime factor responsible for the shortening in subjective duration was the occurrence of the intensity change or the subject's expectancy of this occurrence.

Two variables were analyzed. For the duration discrimination, the level of performance, expressed by an equal variance $d^{\prime}$ index of sensitivity, was measured first by grouping the responses "short" together and "long" together and then by adding the $Z$ transformation of percentages of response "long"/long stimulus and percentages of response "short"/short stimulus. The total probability of responding "short" in each condition was also analyzed (total percentage of responses "short"). For the intensity discrimination, the level of performance, also expressed by the $d^{\prime}$ index, was measured by grouping the responses "weak" together and the responses "strong" together and then by adding the $Z$ transformation of percentages of response "strong"/strong increment and percentages of response "weak"/weak increment. The two $d$ " indices were used to measure the effect of attention on the subjects' performance in the duration and intensity tasks and to observe interference effects. The total percentage of "short" responses provided a measure of underestimation.

\section{EXPERIMENT 1}

The two tasks used in Experiment 1 consisted of identifying both the duration (short or long) of a visual stimulus and a change in its intensity (weak or strong increment), which occurred at various points in time during the stimulus duration. The subjects were expected to shift their attention repeatedly from the temporal to the intensity properties of the stimulus so as to be ready to identify the magnitude of the increment. When the increment took place at the end rather than at the beginning of a trial, more attention shifts were assumed to occur within the course of the trial, and, hence, a greater shortening in judged duration was expected.

\section{Method}

Subjects. Sixteen subjects ( 8 males, 8 females), $20-29$ years of age, took part in this experiment.

Apparatus. The subjects were seated in a dimly lit soundproof room. They faced a video screen on which a green diode appeared with variable duration (short or long) and during which one transient increment (weak or strong) in the luminous intensity occurred at random locations. Four response keys were available, two for each hand ("short" or "long" for the duration, and "weak" or "strong" for the intensity). The experiment was controlled by a microcomputer (HP Vectra QS 165).

Procedure. The task consisted of identifying both the magnitude of the increment and the duration of the green diode. There were four conditions defined by combining weak/strong increment with short/long duration. These four conditions were partially crossed with five attentional allocation instructions. The five instructions specifying how the subject's attention was to be divided between the two tasks were presented on the screen for $5 \mathrm{sec}$ prior to each trial as follows: Condition Id, maximum intensity/ minimum duration; Condition Di, maximum duration/minimum intensity; Condition $h$ h, half/half; Condition D, duration only; Condition $I$, intensity only.

In Conditions Id, $\mathrm{Di}$, and hh, the subjects gave two judgments (one regarding intensity, and the other regarding duration in whatever order); in the last two control conditions (D and I), only one 
judgment was required. One second after the instruction had disappeared from the screen, the green diode was presented. Its duration was $2.5 \mathrm{sec}$ (short duration) or $3.5 \mathrm{sec}$ (long duration). The initial intensity was $0.3 \mathrm{mcd}$ (millicandela) ${ }^{1}$; the intensity of the increment was either $1.4 \mathrm{mcd}$ (weak increment) or $2.2 \mathrm{mcd}$ (strong increment). The increment lasted $100 \mathrm{msec}$. In order to have the same parameters in all trials for Control Conditions I and D and thus to avoid a processing of irrelevant parameters, a single increment of $1.8 \mathrm{mcd}$ (medium increment) was used under the durationonly condition and a single duration of $3 \mathrm{sec}$ (mid-duration) under the intensity-only condition. The subjects responded at the end of the trial by pressing the appropriate keys.

The session contained 320 trials separated in four blocks of 80 . One block consisted of 16 different stimulus combinations [ 3 conditions of attention manipulation $\times 2$ durations $\times 2$ intensities of increment) +4 control conditions (short duration, long duration, weak increment, and strong increment)], each one presented five times. Thus, in total, each stimulus combination was presented 20 times. The increment was given various locations in the course of the stimulus. For each stimulus combination, increments were distributed such that it occurred seven times in the first part of the stimulus, six times in the second part, and seven times in the third part. The stimulus was divided into three equal parts, but without including its initial and last $0.5 \mathrm{sec}$. Thus, increments could occur between 0.5 and $2 \mathrm{sec}$ for the short duration (Parts 1, 2, and 3 were, respectively, $0.5-1,1-1.5$, and $1.5-2 \mathrm{sec}$ ) and between 0.5 and $3 \mathrm{sec}$ for the long duration (Parts 1, 2, and 3 were, respectively, $0.5-1.3,1.3-2.2$, and $2.2-3 \mathrm{sec}$ ). In each part, the location of the increment was randomized.

Prior to the test trials, the subjects were given two training sessions: (A) intensity training, and (B) duration training, (in counterbalanced order). The first step consisted of the presentation of five pairs of stimuli: weak-strong in A ( $1.4 \mathrm{or} 2.2 \mathrm{mcd}, 3 \mathrm{sec}$ ) and short-long in $B(2.5$ or $3.5 \mathrm{sec}, 1.8 \mathrm{mcd})$. Intervals of $1 \mathrm{sec}$ within a pair and of $2 \mathrm{sec}$ between pairs were used. In a second step, the subject performed 20 trials ( 10 weak and 10 strong in $A, 10$ short and 10 long in B, randomly presented), with 5 -sec intertrial intervals. After each response (press on the appropriate button), knowledge of results was delivered: The words right or wrong appeared on the screen. In a third step, there were 50 trials without knowledge of results ( 25 for intensity and 25 for duration). The performance obtained in each of these single tasks was called out-control to distinguish it from the control conditions (D or I) included in the experimental session (where these single tasks were mixed up with dual tasks within a block of trials). The out-control blocks of trials were analyzed to check whether the increment position had an effect on judgments even when no identification of intensity was required in any trial of the block.

The stimulus parameters were based on pilot studies designed to yield $70 \%-90 \%$ correct judgments in the single-task conditions.

\section{Results $^{2}$}

$\boldsymbol{d}^{\prime}$ index. Figure 1a gives the $d^{\prime}$ index as a function of the attentional conditions in the duration and intensity tasks. It shows that as $d^{\prime}$ increased for one task, it decreased for the other. Thus, performance is related to the explicit instructions regarding the allocation of attention. As it is illustrated in Figure $1 b$, the $d^{\prime}$ index in the duration task was higher when the increment took place in the first or second parts of the stimulus than when the increment took place in the third part.

Percentage of "short" responses. Figure 2a shows the percentage of "short" responses as a function of attention conditions (i.e., in the D, Di, hh, and Id conditions). This percentage increased when more attention was di-
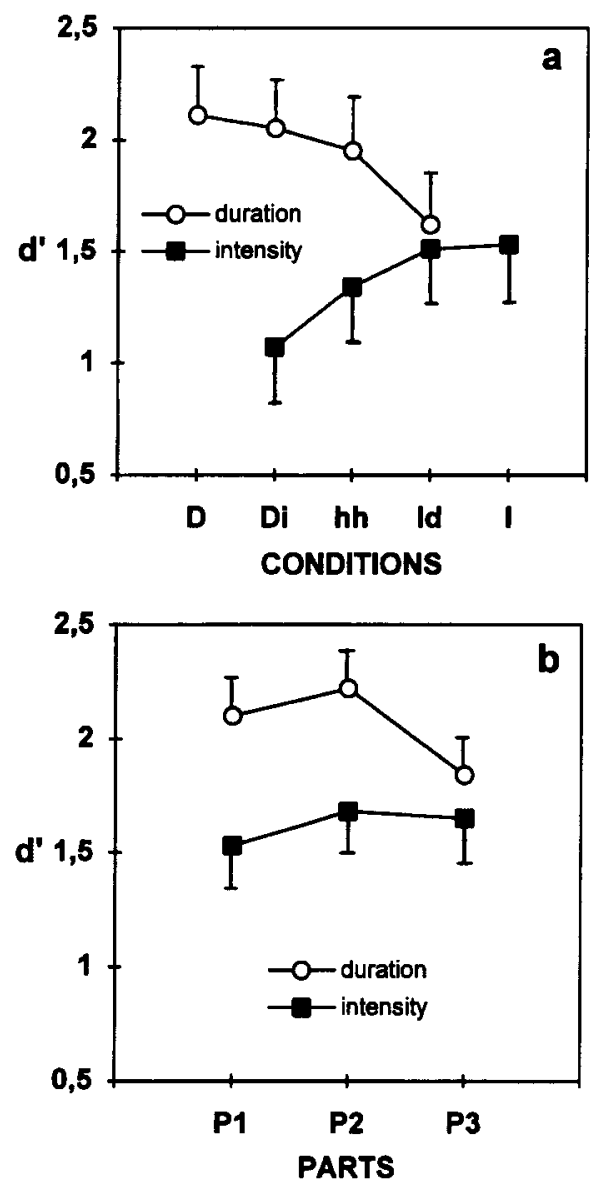

Figure 1. Performance levels $\left(d^{\prime}\right)$ in the duration and intensity tasks as a function of the attentional conditions (a) and of the increment position (b) in Experiment 1 (averaged data, 16 subjects). In Panel a, on the abscissa, D, Di, hh, Id, and I refer to duration only, maximum duration/minimum intensity, half/half, maximum intensity/minimum duration, and intensity only, respectively. In Panel b, P1, P2, and P3 refer to the first, second, and third parts of the stimulus. Error bars reflect $95 \%$ confidence intervals around each mean (according to Loftus and Masson's, 1994, procedure for computing confidence intervals in within-subject designs).

rected toward the discrimination of intensity (from $D$ to Id). Figure $2 b$ illustrates the effect of position: As expected, the percentage of "short" responses increased when the increment occurred later in the trial.

Another analysis was performed on the percentage of "short" responses obtained in the out-control block of trials of the duration task, in order to check whether the increment position influenced performance when no intensity judgment was required. The percentage of "short" responses was higher when the increment appeared in the last third of the stimulus (Part $1[\mathrm{P} 1]=38.9 \%$, Part 2 $[\mathrm{P} 2]=52.7 \%$, Part $\left.3[\mathrm{P} 3]=63.4 \%, M S_{\mathrm{e}}=3.02\right)$.

\section{Discussion}

$d^{\prime}$ index. The $d^{\prime}$ indexes indicated that performance on each task varied with the attentional conditions. These 

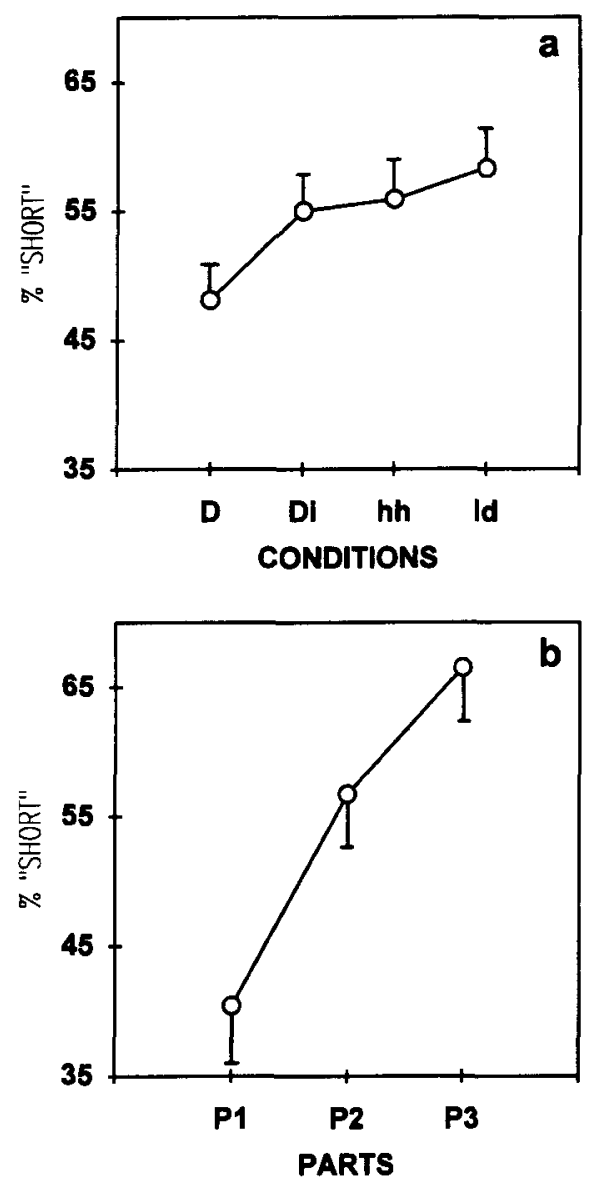

Figure 2. Percentage of "short" responses as a function of the attentional conditions (a) and of the increment position (b) in Experiment 1 (averaged data, 16 subjects). D, Di, hh, and Id refer to duration only, maximum duration/minimum intensity, half/half, and maximum intensity/minimum duration, respectively. $\mathbf{P 1}, \mathbf{P 2}$, and $P 3$ refer to the first, second, and third parts of the stimulus. Error bars reflect $95 \%$ confidence intervals around each mean (according to Loftus and Masson's, 1994, procedure for computing confidence intervals in within-subject designs).

data confirm the results obtained by Macar et al. (1994) with different nontemporal tasks. The observed pattern of interference between the two tasks supports the assumption that these tasks competed for the same attentional resources and is in accord with the models of time perception proposed by Thomas and Weaver (1975) and Zakay (1989).

Furthermore, in the duration task, the $d^{\prime}$ index depended on the position of the intensity change. Sensitivity was higher when the increment occurred in the first or second part of the stimulus than when it occurred in the third part. One possible explanation rests on the conditional probability of the increment occurrence. In reaction time tasks, it has been shown that the subject's level of motor preparation increases with the conditional probability of the response signal, which is maximal at the end of the trial when the signal has not yet occurred (Durup $\&$ Requin, 1970). Accordingly, it is likely that, in the pres- ent experiment, the subjects devoted more attention to the intensity task as time elapsed. As a result, the performance in the duration task was most impaired when the increment occurred late.

Percentage of "short" responses. The percentage of "short" responses increased as the subject's attention was directed toward the intensity task as a result of the instructions. This result lends further support to the hypothesis of an inverse relationship between judged duration and the amount of attention accorded to nontemporal information.

Furthermore, the decrease in judged duration was most marked when the increment occurred in the last part of the trial - that is, when its conditional probability of occurrence was highest. This suggests again that, as the trial proceeded, attention to the intensity task became more prominent. In line with the timer model, we propose that attention shifts disrupted the accumulation of pulses underlying temporal processing.

The effect of increment position obtained in the outcontrol block of trials of the duration task is surprising since no intensity judgment was required; hence, the subject did not need to allocate attention to the processing of intensity. Two explanations are possible. The simpler one is that the subjects shifted their attention to the intensity task because they expected the increment even though it was irrelevant. Attention shifts might be more numerous when the increment took place in the last part of the stimulus because of the conditional probability effect. The number of attentional shifts was likely smaller, nevertheless, than in the other attention conditions, in which the subjects had to process intensity. Accordingly, the percentage of "short" responses was higher in the latter cases. This effect remains surprising, however, since the subjects always performed the out-control block of trials before the experimental block and, hence, did not know that a dual task would be required thereafter.

An alternative explanation is that the occurrence of an increment automatically causes a shift in attention, whether the subjects did or did not need to attend to it. This might produce a resetting of the timer, or a loss of part of the pulses that had been accumulated before the occurrence of the increment. If the loss is greater when the accumulator contains more pulses, this could explain why, even in the out-control block of trials, subjective duration was shorter when the increment occurred later.

This idea could, of course, also apply in the dual-task conditions. Thus, it raises a new question: Which is the most important cause of the reduction in subjective duration due to increment position? Is it an increase in the amount of the attention shifts due to expectancy of the increment, or is it an automatic orienting of attention due to increment occurrence? This question will be examined in Experiment 2.

\section{EXPERIMENT 2}

In Experiment 2, a small proportion of trials containing no increment were added. The subjects were told that the increment would take place in most trials. Two hy- 
potheses were contrasted: (1) If the orienting of attention due to the detection of the increment is responsible for the shortening in subjective duration, this duration should be shorter in the trials with increment than in the noincrement trials. (2) If the attention shifts due to the expectancy created by the instructions are responsible for the shortening in subjective duration, this duration should be longer in the trials with increment than in the no-increment trials. Indeed, the no-increment trials, because they are mixed with a large proportion of other trials, should not alter the subject's expectancy. Hence, they would not only generate attention shifts, but they would also make the shifts particularly persistent since no increment would appear.

The percentage of "short" responses is the key measure to evaluate these hypotheses. Only this measure is presented below.

\section{Method}

Subjects. Twelve subjects ( 6 males, 6 females, different from those in Experiment 1), 20-29 years of age, participated in this experiment.

Procedure. The procedure was identical to that used in Experiment 1 , except that trials without an intensity increment were included. The stimuli were 2.5 and $3.5 \mathrm{sec}$ for the short and long durations, respectively. The basic level of intensity was $0.9 \mathrm{mcd}$, and the intensities of the increments were $1.2 \mathrm{mcd}$ (small) and $2.4 \mathrm{mcd}$ (large). Similar to in Experiment 1, a mid-duration of $3 \mathrm{sec}$ was used in the intensity-only condition and a mid-intensity of $1.8 \mathrm{mcd}$ in the duration-only condition. One session contained 384 trials performed in four blocks of 24 different conditions of stimulation, each one presented four times. These 24 conditions corresponded to three attentional instructions ( $\mathrm{Di}, \mathrm{hh}, \mathrm{Id}) \times 2 \mathrm{du}-$ rations (short or long) $\times 3$ cases of increments (weak, strong, or no increment), plus 6 control conditions. The 6 control conditions were divided in 4 duration-only conditions in which the subjects judged only the duration of the stimulus (short duration and medium increment, long duration and medium increment, short duration and no increment, long duration and no increment) and 2 intensity-only conditions in which only intensity judgments were required (mid-duration and strong increment, mid-duration and weak increment). Therefore, each attentional condition included $67 \%$ trials with an increment and $33 \%$ without; in the durationonly condition, the proportions were $50 \%-50 \%$.

The training session was similar to that of Experiment 1.

\section{Results}

Figure 3a illustrates the percentage of "short" responses as a function of the attentional conditions. The more attention was allocated to intensity, the higher was the percentage of "short" responses. The effect of the factor of position (including the no-increment case) is shown on Figure $3 b$. The percentage of "short" responses was found to vary both with the presence or absence of the increment and with the increment position. When no increment took place, this percentage was higher than when the increment occurred in the first part of the stimulus, but lower than when it occurred in the third part.

In the out-control block of trials, the moment of occurrence of the increment and its presence or absence had no effect on subjective duration ( $\mathrm{P} 1=47.9 \%, \mathrm{P} 2=54.16 \%$, $\mathrm{P} 3=43.1 \%$, no increment $=47.8 \%, M S_{\mathrm{e}}=4.02$ ).
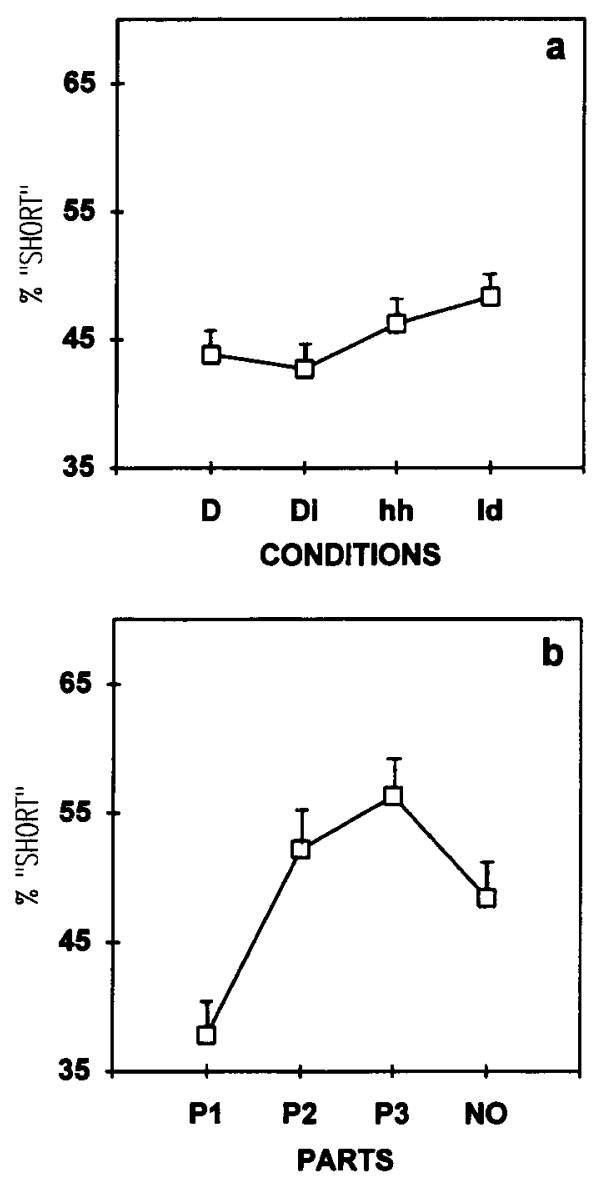

Figure 3. Percentage of "short" responses as a function of the attentional conditions (a) and of the increment position (b) in Experiment 2 (averaged data, 12 subjects). D, Di, hh, and Id refer to duration only, maximum duration/minimum intensity, half/ half, and maximum intensity/minimum duration, respectively. P1, P2, and $P 3$ refer to the first, second, and third parts of the stimulus. Error bars reflect $95 \%$ confidence intervals around each mean (according to Loftus and Masson's, 1994, procedure for computing confidence intervals in within-subject designs).

\section{Discussion}

As shown by the percentage of "short" responses, there was a shortening in subjective duration when no increment occurred. This result demonstrates that the attention shifts due to the expectancy of a probable event were the prime factor determining subjective duration in the dual-task paradigm. Attentional shifts to the intensity task increased until the end of the trial, since the subjects were expecting the intensity increment to take place. However, when the increment did occur, the highest percentage of "short" responses was obtained if it was placed in the last part of the stimulus. Thus, in addition to the effect of attention shifts due to expectancy, there was an effect of the increment occurrence itself that caught the subjects' attention. In line with the timer hypothesis, it can be postulated that this processing of the increment produced an additional gap in pulse accu- 
mulation. The joint effects of expectancy and of detection were particularly obvious when the probability of increment occurrence was high.

Note that the orienting of attention induced by this occurrence cannot be considered simply an automatic effect. This is shown by the data obtained in the durationonly condition. Here, the percentage of "short" responses was influenced neither by the presence or absence of the increment nor by its moment of occurrence. Thus, when half of the trials in a block contained no increment, the subjects were able to ignore the increment in the other trials and to avoid shifting their attention to intensity.

Taken together, these data suggest that the accumulation of pulses by the timer can be transiently interrupted by attentional shifts that are due to both the expectation and the detection of a target and that are very dependent on the target conditional probability.

\section{GENERAL DISCUSSION}

The dual-tasks used here confirm the existence of interference effects between a temporal task and a nontemporal task and show that the subjective duration of a stimulus decreases when attention is allocated to the nontemporal properties of that stimulus. These results, which confirm those we reported previously (Macar et al., 1994), are congruent with the attentional model of time perception (Thomas \& Weaver, 1975; Zakay, 1989), and they emphasize the importance of attentional resources on the functioning of the internal timer.

The underestimation is a strong prediction of the attentional model based on the concept of a timer that accumulates pulses as a function of time and loses a certain number of pulses when attention is disrupted. A priori, other possibilities exist when there are interference effects: overestimation, like underestimation, can be expected. Overestimation would refute a cumulative timer but would be consistent with the idea that temporal judgments depend on the amount of processed nontemporal information. This idea seems tenable in the case of retrospective judgments of time, which are not appropriate to activate a cumulative timer. Under retrospective conditions, a time judgment is required after a given period, without any prior warning, and subjects' attention is directed toward other parameters of the task. In contrast, under prospective conditions, subjects are forewarned that a temporal judgment will be required. Numerous studies have shown that overestimation occurs in retrospective situations, whereas underestimation occurs in prospective situations, as used here (Hicks et al., 1976; Zakay, 1989).

The results also show that interference effects can be obtained when the two tasks involve two different aspects of one single stimulus and are not limited to the case of two different stimuli. These interference effects suggest that the two tasks used the same attentional resources and that these resources are limited (Bonnel, Possamai, \& Schmitt, 1987; Navon \& Gopher, 1979; Posner \& Petersen, 1990). According to Kramer and Spinks (1991), the fact that subjects can perform different tasks simulta- neously and give priority to one task to the detriment of the other emphasizes that limited resources can be divided and allocated in a flexible manner.

The use of an instructional manipulation to vary how subjects allocate attentional resources confirms the importance of controlled attention in temporal processing (Jackson, 1985; Macar et al., 1994). Subjects can voluntarily allocate attention to the processing of temporal information, as with other types of information. This is congruent with the hypothesis that stimulus duration is processed in itself rather than being inferred from other stimulus dimensions.

An important question is to determine whether the effects of attention take place at an early or late phase of temporal processing. The information-processing model of timer proposed by Gibbon et al. (1984) contains three interrelated parts: the clock, the memory, and the decision stages. The clock consists of a pacemaker, which produces pulses at a given rate, and a switch, which is driven by the external stimuli and with a certain latency controls the access to an accumulator, which sums up the pulses. A comparator contrasts the value stored in the accumulator with the values that have been stored in reference memory on previous trials. The response is determined on the basis of this comparison.

Each stage of this model can be a source of variance, and the effects of attention may a priori affect any or all of these stages (Church, 1984). Our data suggest that the disruption of attention, whether due to stimulus detection or expectancy, impairs the processing of duration. We propose that the shortening in subjective duration due to the attentional instructions reflects a diminution in the accumulated pulses, which may concern the access of the pulses to the accumulator.

At an early stage, fewer pulses might be accumulated because attentional shifts act on the switch, which would block the access to the accumulator. Such an effect has been suggested by studies on animals in various temporal conditioning schedules (Church, 1978; Roberts, 1981). For instance, rats were trained to discriminate the duration of continuous signals and then tested with signals including gaps. Their performance revealed that they could time the duration that the stimulus was on and ignore the duration that the stimulus was off. This suggests that when a signal made up of $2 \mathrm{sec}$ "on," $1 \mathrm{sec}$ "off," and again $2 \mathrm{sec}$ "on" is presented, pulse accumulation takes place during the first stimulus segment, is blocked during the gap, and resumes during the second stimulus segment. Thus, performance with a signal including a $1-\mathrm{sec}$ gap was similar to performance with a signal that was $1 \mathrm{sec}$ shorter. Other data concerned with switch latency (Meck, 1984) also indicate that the switch is sensitive to attention effects. Zakay and Block (1995) proposed that the effects of attention are mediated by a "gate" located between the pacemaker and the switch. The gate would open wider, and more pulses emitted by the pacemaker would pass through to the accumulator when attention is allocated to time. Our data, however, are easier to explain by considering an effect on the switch within the course 
of the stimulus than by considering an effect on a gate functioning before the stimulus is presented.

An alternative possibility that might account for the shortening in subjective duration we observed concerns pulse storage: When attention is disrupted, part of the pulses stored in the accumulator might be lost. The present data do not enable us to decide between these hypotheses. This issue is an exciting challenge for further research.

\section{REFERENCES}

Bonnel, A. M., Possamai, C. A., \& Schmitt, M. (1987). Early modulation of visual input: A study of attentional strategies. Quarterly Journal of Experimental Psychologv, 39A, 757-776.

CAsini, L., MACAR, F., \& Grondin, S. (1992). Time estimation and attentional sharing. In F. Macar, V. Pouthas, \& W. Friedman (Eds.), Time, action and cognition: Towards bridging the gap (pp. 177180). Dordrecht: Kluver.

Сhurch, R. M. (1978). The internal clock. In S. H. Hulse, H. Fowler, \& W. K. Honig (Eds.), Cognitive processes in animal behavior (pp. 227-310). Hillsdale, NJ: Erlbaum.

ChURCH, R. M. (1984). Properties of the internal clock. In J. Gibbon \& L. Allan (Eds.), Timing and time perception (Annals of the New York Academy of Sciences, Vol. 423, pp. 566-582). New York: New York Academy of Sciences.

Creelman, C. D. (1962). Human discrimination of auditory duration. Journal of the Acoustical Society of America, 34, 582-593.

DURUP, H., \& REQUIN, J. (1970). Hypothèses sur le rôle des probabilités conditionnelles du signal d'exécution dans le temps de réaction simple [Hypotheses concerning the role of conditional probabilities of the go signal on simple reaction times]. Psychologie française, $15,37-46$.

Gibbon, J., Church, R. M., \& Meck, W. H. (1984). Scalar timing in memory. In J. Gibbon \& L. Allan (Eds.), Timing and time perception (Annals of the New York Academy of Sciences, Vol. 423, pp. 52-77). New York: New York Academy of Sciences.

Grondin, S., \& MACAR, F. (1992). Dividing attention between temporal and nontemporal tasks: A performance operating characteristic POC - analysis. In F. Macar, V. Pouthas, \& W. Friedman (Eds.), Time, action and cognition: Towards bridging the gap (pp. 119-128). Dordrecht: Kluver.

Hicks, R. E., Miller, G. W., \& Kinsbourne, M. (1976). Prospective and retrospective judgements of time as a function of amount of information processed. American Journal of Psychology, 89, 719-730.

$\mathrm{J}_{\mathrm{ACKSON}}$ J. L. (1985). Is the processing of temporal information automatic or controlled? In J. A. Michon \& J. L. Jackson (Eds.), Time, mind and behavior (pp. 179-190). Heidelberg: Springer-Verlag.

Kahneman, D. (1973). Attention and effort. Englewood Cliffs, NJ: Prentice Hall.

Kramer, A., \& SPINKS, J. (1991). Capacity views of human information processing. In J. R. Jennings \& M. G. H. Coles (Eds.), Handbook of cognitive psychophysiology: Central and nervous system approaches (pp. 179-248). Chichester, U.K.: Wiley.

LOFTUS, G. R., \& MASSON, M. E. J. (1994). Using confidence intervals in within-subject designs. Psychonomic Bulletin \& Review, 1, 476-490.
MaCAR, F., Grondin, S., \& Casini, L. (1994). Controlled attention sharing influences time estimation. Memory \& Cognition, 22, 673-686.

MCCLAIN, L. (1983). Interval estimation: Effect of processing demands on prospective and retrospective reports. Perception \& Psychophysics, 34, 185-189.

MECK, W. H. (1984). Attentional bias between modalities: Effect on the internal clock, memory, and decision stages used in animal time discrimination. In J. Gibbon \& L. Allan (Eds.), Timing and time perception (Annals of the New York Academy of Sciences, Vol. 423. pp. 528-541). New York: New York Academy of Sciences.

Miall, R. C. (1992). Oscillators, predictions and time. In F. Macar, V. Pouthas, \& W. J. Friedman (Eds.), Time, action and cognition: Towards bridging the gap (pp. 215-227). Dordrecht: Kluwer.

Navon, D. (1985). Attention division or attention sharing? In M. I. Posner \& O. M. Marin (Eds.), Attention and performance XI (pp. 88-99). Hillsdale, NJ: Erlbaum

NAVON, D., \& GoPHER, D. (1979). On the economy of the humanprocessing system. Psychological Review, 86, 214-255.

Posner, M. I., \& Petersen, S. E. (1990). The attention system of human brain. Annual Review of Neurosciences, 13, 25-42.

ROBERTS, S. (1981). Isolation of an internal clock. Journal of Experimental Psychology: Animal Behavior Processes, 7, 242-268.

SPERLING, G., \& MELCHNER, M. J. (1978). The attention operating characteristic: Examples from visual search. Science, 202, 315-318.

Thomas, E. A. C., \& Weaver, W. B. (1975). Cognitive processing and time perception. Perception \& Psychophysics, 17, 363-367.

Treisman, M. (1963). Temporal discrimination and the indifference interval: Implications for a model of the "internal clock." Psychological Monographs (Whole No. 576).

Treisman, M. (1993). On the structure of the temporal sensory system. Psychologica Belgica, 33, 271-284.

ZAKAY, D. (1989). Subjective and attentional resource allocation: An integrated model of time estimation. In I. Levin \& D. Zakay (Eds.), Time and human cognition (pp. 365-397). Amsterdam: NorthHolland.

ZAKAY, D. (1992). On prospective time estimation, temporal relevance and temporal uncertainty. In F. Macar, V. Pouthas, \& W. J. Friedman (Eds.), Time, action and cognition: Towards bridging the gap (pp. 109118). Dordrecht: Kluwer.

ZAKAY, D., \& BLOCK, R. (1995). An attentional-gate model of prospective time estimation. In M. Richelle, V. De Keyser, G. d'Ydewalle, \& A. Vandierendonck (Eds.), Time and the dynamic control of behavior (pp. 167-178). Liège: Presses de l'Université.

\section{NOTES}

1. One candela $=1$ lumen/steradian. The luminous intensity is proportional to electric intensity and, here, was worth $3 \mathrm{mcd}$ for $20 \mathrm{~mA}$

2. Results concerning the percentage of "weak" responses, which were reported in our previous paper (Macar et al., 1994) are available from the authors, as are all the analyses of variance related to the present results.

(Manuscript received April 5, 1996; revision accepted for publication November 26, 1996.) 